\title{
Determining the performance of alfalfa population collected from a narrow agroeceological zone of Turkey
}

\section{Erdal Cacan ${ }^{1} \odot$ Kagan Kokten $^{1} \odot$ Seyithan Seydosoglu ${ }^{2 *} \odot$}

${ }^{1}$ University of Bingöl Faculty of Agricultural Department of Field Crops, Bingöl-Turkey.

${ }^{2}$ University of Siirt Faculty of Agricultural Department of Field Crops, Siirt-Turkey. E-mail: seyithanseydosoglu@siirt.edu.tr. "Corresponding author.

ABSTRACT: This study was carried out to determine the yield and quality characteristics of some alfalfa village populations cultivated by producers in Bingol province of Turkey. Alfalfa seeds were obtained from 23 different locations in 2015. A three replicated, randomised complete block designed field trial was established in 2016 including these genotypes and four registered varieties. As a result of three year trials, it was determined that the populations obtained from the villages of Servi, Mutluca, Garip, Sarlçiçek-1 and Bagliisa produced highest green herbage and hay yields. When we compare the quality results, Kumgecit, Küçüktekören and Şenköy-1 populations stands out in terms of crude protein ratio. Highest crude protein yield was obtained from Servi population. In terms of relative feed value, it was observed that the varieties were better qualfied than the populations. It is concluded that the populations of Servi, Mutluca, Garip, Sarıçiçek-1 and Bagliisa can be used in breeding studies especially to obtain genetic progress in yield of alfalfa for feed and bioenergy production. For the genetic improvement of current genotypes with higher crude protein ratio, Kumgecit, Küçuktekören and Senköy-1 populations can be used as a source. This study showed that, East Anatolia region is not just live-conserving highly diversified species but also covering special intra-species genetic diversity in microclimatic zones of Turkey to be used to improve the global forage and bioenergy cropproduction.

Key words: Medicago sativa L. alfalfa, population, yield, quality, Anatolia.

Determinação da população de alfafa coletada em uma estreita zona agroecológica da Turquia

RESUMO: Este estudo foi realizado para determinar as características de rendimento e qualidade de algumas populações de alfafa cultivadas por produtores na província de Bingol, na Turquia. As sementes de alfafa foram obtidas de 23 locais diferentes em 2015. Em 2016, foi estabelecido um ensaio de campo com três repetições, aleatórias e desenvolvido em bloco, incluindo esses genótipos e quatro variedades registradas. Com o resultado de testes de três anos, determinou-se que as populações obtidas nas aldeias de Servi, Mutluca, Garip, Sarıçiçek-1 e Bagliisa produziam maiores colheitas de forragem verde e feno. Quando comparamos os resultados de qualidade, as populações Kumgecit, Küçüktekören e Şenköy-1 se destacam em termos de proporção de proteina bruta. O maior rendimento de proteina bruta foi obtido da população de Servi. Em termos de valor relativo de ração, observou-se que as variedades eram mais qualificadas do que as populações. Conclui-se que as populações de Servi, Mutluca, Garip, Sarlçiçek-1 e Bagliisa podem ser utilizadas em estudos de melhoramento, especialmente para obter progresso genético no rendimento de alfafa para produção de ração e bioenergia. Para o aprimoramento genético dos genótipos atuais com maior proporção de proteína bruta, as populações Kumgecit, Küçüktekören e Senköy-1 podem ser usadas como fonte. Este estudo mostrou que, a região da Anatólia Oriental não é apenas espécies altamente diversificadas conservadoras de vida, mas também abrange diversidade genética intraespécie especial em zonas microclimáticas da Turquia, a ser usada para melhorar a produção global de forragem e bioenergia. Palavras-chave: Medicago sativa alfafa, população, rendimento, qualidade, Anatólia.

\section{INTRODUCTION}

Alfalfa (Medicago sativa L.) is a widely cropped legume forage for hay, pasture and silage production throughout the World (LI \& BRUMMER, 2012). It provides significant habitat to a wide range of wildlife, including beneficial insects. It improves yields and reduces nitrogen fertilizer needs for subsequent crops. Alfalfa also helps to remediate soil and water contamination (PUTNAM et al., 2001).
Alfalfa also has considerable potential as a feedstock for production of ethanol due to its high biomass production, perennial nature, ability to provide its own nitrogen fertilizer and valuable co-products. Its stems are an excellent feedstock for cellulosic ethanol via fermentation or gasification (MARTIN et al., 2010). The stems has a potential as biomass energy crops especially in temperate regions where their bioconversion potential is high as an energy crop (DIEN et al., 2006). Alfalfa has been considered as 
a biofuel feedstock where a system has been proposed to produce electricity from the stems following utilization of the leaves as a livestock feed (SHEAFFER, 2000).

Increasing forage yield is main priority for most alfalfa breeding programs, but yield trends has stagnated over the past two decades (RIDAY \& BRUMMER, 2002). Currently, alfalfa breeding relies on recurrent phenotypic selection (LI \& BRUMMER, 2012). Alfalfa cultivars are synthetic populations formed from 8 to 200 parents and have a broad genetic base. In the study of JULIER et al. (2000), when intra-cultivar variation was compared with amongcultivars for energy value traits, morphological traits and dry matter yield, variance accounted for 31 to $70 \%$ of the genetic variance for leaf-to-stem ratio and quality traits and 57 to $100 \%$ for morphological traits and dry matter yield. Researches informed that large intracultivar variation for yield-related traits could impart yield stability across environments by competition in alfalfa canopies and cultivar variation could be an additional source of genetic variation in breeding programs for quality traits to achieve a higher genetic gain per breeding cycle (JULIER et al., 2000).

The most cultivated feed crop in Turkey is also alfalfa. Since this species is perrennial, well adapted to different conditions, moweable multiple times in a single year vegetation period, good rotation crop, in between with its high hay yield and quality (SOYA et al., 2004; AVCIOGLU et al., 2009).

SARUHAN \& KUSVURAN, (2011) determined that the yield performance of some alfalfa genotypes in Southeast Anatolia Region conditions of Turkey was at good levels. They determined green grass yield at 35.1-19.0 t/ha, hay yield at 9.4-12.6 $\mathrm{t} / \mathrm{ha}$, the ratio of crude protein at 17.9-22.3\%. To determine the yield of some alfalfa genotypes in Mediterranean climatic conditions in Turkey, KAVUT et al. (2014) carried out a field trial and obtained 95.2-118.9 t/ha green grass yield, and 20.3-27.1 t/ha hay yield. In the study of OTEN \& ALBAYRAK (2018), which examined the quality characteristics of 26 alfalfa genotypes collected from South-West natural flora of Anatolia, the average crude protein ratio was $15.8 \%$, the ADF (acid detergent insoluble fiber) rate was $36.7 \%$ and the NDF (neutral detergent insoluble fiber) rate was $47.2 \%$.

In other important grass production zone of Turkey, in East Anatolia, alfalfa is covering 7.392 ha solely in Bingol province, with 292.000 tons production and 39.5 t/ha green grass yield (TURKSTAT, 2019). Alfalfa hay yield differs among regions in Turkey where is is 2.5-3.0 t/ha in rainfed, 5.0-25.0 t/ha in irrigated conditions (SOYA et al., 2004; AVCIOGLU et al., 2009). Since the main source of income in Bingol province of Turkey is animal husbandry, alfalfa cultivation has a long history. Alfalfa cultivation in Bingol is mostly carried out by population seeds.

The purpose of this study was to determine the yield and quality characteristics of some alfalfa populations in Bingol with the aim to utilise them in breeding programmes.

\section{MATERIALS AND METHODS}

Research field was located in Bingöl province, Genc subprovince of Turkey. Altitude of the field was $986 \mathrm{~m}$ and located at 38.749460 North latitude and 40.536780 East longitude coordinates. In 2015 , seeds of local alfalfa populations were obtained from farmers in the villages of Bingol. A total of 23 local genotypes were collected.

The trials was conducted in Bingol University research fields. The research area is located at 38.749460 North latitude and 40.536780 East longitude coordinates (Figure 1). Long term (19902015) average temperature is $12.3{ }^{\circ} \mathrm{C}$, precipitation is $917.8 \mathrm{~mm}$ and humidity is $56.6 \%$ in Bingol. July and August are the months with highest temperature with lowest rainfall and humidity. Most of the precipitation realises in winter period (Table 1).

Long yers (1961-2018) average climatic records obtained from Bingöl Provincial Meteorological Centerindicates that average air temperature of the trial location is $12.1{ }^{\circ} \mathrm{C}$ and precipitation is $943.3 \mathrm{~mm}$ (ANONYMOUS, 2019).

Soil samples obtained from trial field were analysed in University of Bingol Soil Analysis Laboratory. For the evaluation of the analysis results, limit values determined by of SEZEN (1995) was used as base. Soil of research area is sandy-clayey-loam structured (59.5\% sand, $18.2 \%$ clay, $22.3 \%$ loam), has neutral $\mathrm{pH}$ ratio (7.26), medium organic matter $(2.1 \%)$, low phosphorus $(51 \mathrm{~kg} / \mathrm{ha})$, low potassium $(436 \mathrm{~kg} / \mathrm{ha})$ and low salt contents $(0.34 \mathrm{mS} / \mathrm{cm})$.

For comparison of 23 genotypes, four varieties (Basbag, Bilensoy 80, Savas and Gea) were used as reference.

Field trial was established on date 06.04.2016 with three replications according to randomized block design. The length of each parcel was five meters, each row was consisting of six rows and interrow space was $20 \mathrm{~cm}$. Seeding rate was 30 $\mathrm{kg} / \mathrm{ha}$ of seed were planted by hand in accordance with TURAN (2019). $40 \mathrm{~kg} / \mathrm{ha}$ nitrogen and $100 \mathrm{~kg} /$ ha phosphorus were applied at sowing. Field trial was carried out under irrigtaed conditions. Harvests were 


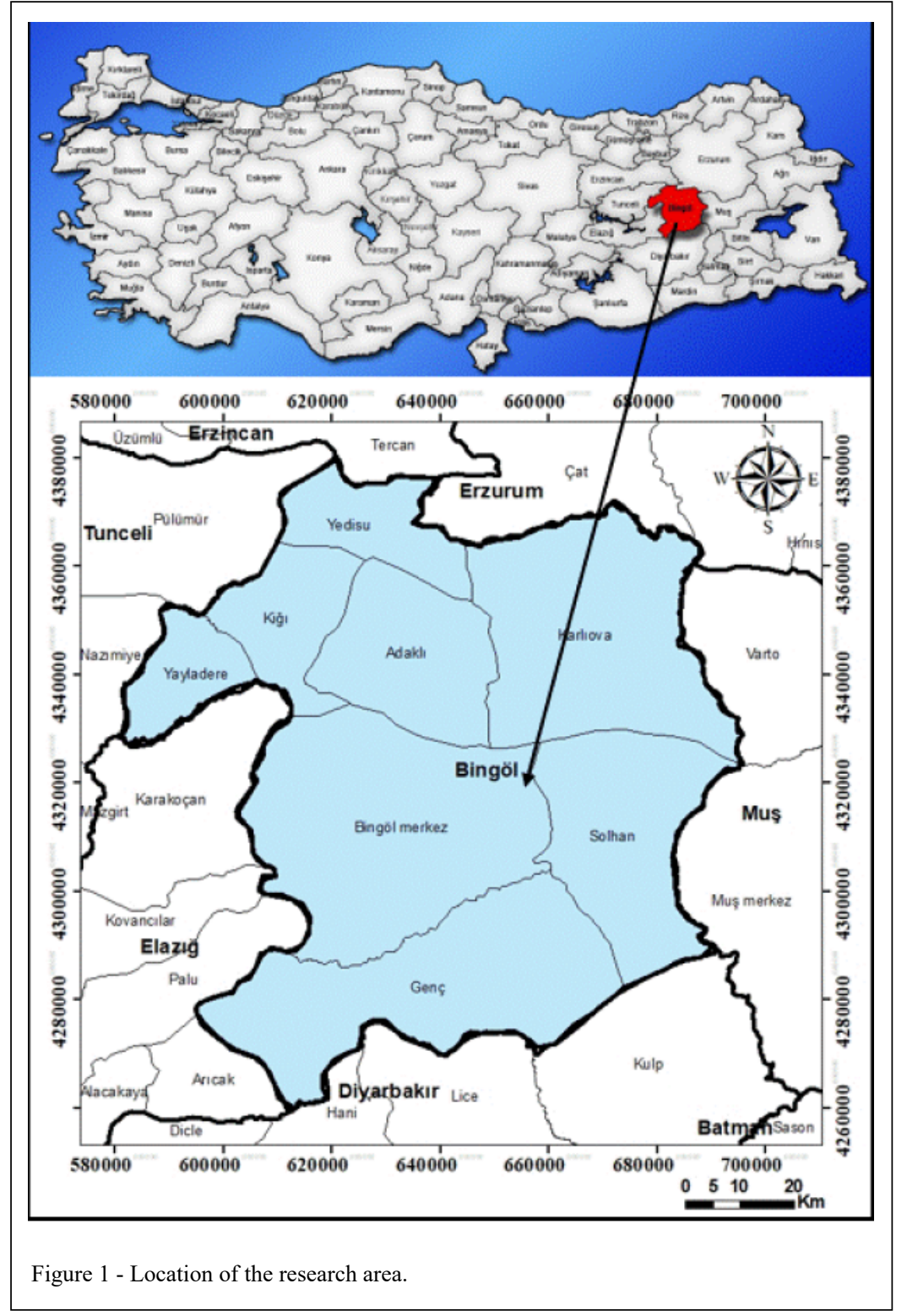

conducted during the $10 \%$ flowering period. Parcels were harvested three times in 2016, four times in 2017 and four times in 2018. Green herbage yield was calculated and followingly 500 grams of green herbage sample was dried at $70{ }^{\circ} \mathrm{C}$ for 48 hours to measure dry yields in accordance with TURAN, (2019). CP (crude protein), ADF (acid detergent insoluble fiber) and NDF (neutral detergent insoluble fiber) ratios were determined by using grinded hay samples by NIRS (Near Infrared Spectroscopy) instrument. Using the obtained values, the digestible dry matter (DDM) and the relative feed value (RFV) were calculated by using given equations 1 and 2 (MORRISON, 2003).
$\mathrm{DDM}=88.9-(0.779 \times \mathrm{ADF})$

(Eq.1)

$\mathrm{RFV}=((\mathrm{DDM} \times \mathrm{DMI}(120 / \mathrm{NDF})) / 1.29 \quad$ (Eq.2) In these equations, DDM=Digestable Dry Matter; RFV=Relative Feed Value; DMI=Dry Matter Intake. JUMP 0.5 statistical program was used for variance analysis and Tukey test was used for comparisons.

\section{RESULTS AND DISCUSSION}

Green herbage and hay yields

Statsitically significant differences $(\mathrm{P}<0.01)$ were obtained for green herbage yields and hay yields based on genotypes and trial yerswhich are

Ciência Rural, v.50, n.11, 2020. 
Table 1 - Bingol Province Meteorological Data.

\begin{tabular}{|c|c|c|c|c|c|c|c|c|c|c|c|c|}
\hline \multirow[t]{2}{*}{ Months } & \multicolumn{4}{|c|}{-------Average Temperature $\left({ }^{0} \mathrm{C}\right)------$} & \multicolumn{4}{|c|}{---------Total Precipitation (mm)-------- } & \multicolumn{4}{|c|}{--------Relative Humidity (\%)------ } \\
\hline & 2016 & 2017 & 2018 & LY & 2016 & 2017 & 2018 & LY & 2016 & 2017 & 2018 & LY \\
\hline January & -2.8 & -3.7 & 2.0 & -2.5 & 235.1 & 63.9 & 204.0 & 154.0 & 75.3 & 71.1 & 72.7 & 73.3 \\
\hline February & 2.4 & -2.3 & 5.2 & -0.9 & 86.3 & 32.9 & 74.9 & 137.7 & 73.7 & 61.6 & 65.8 & 72.2 \\
\hline March & 7 & 5.9 & 10.3 & 4.9 & 125.5 & 114.5 & 72.2 & 124.1 & 60.4 & 64.7 & 59.1 & 64.2 \\
\hline April & 13.9 & 10.8 & 14.4 & 10.9 & 45.5 & 166.4 & 57.1 & 103.8 & 48.4 & 58.8 & 44.1 & 61.2 \\
\hline May & 16.3 & 16.4 & 16.4 & 16.2 & 62.2 & 92.4 & 163.0 & 66.8 & 57.4 & 56.2 & 67.9 & 55.8 \\
\hline June & 22.2 & 22.6 & 22.6 & 22.6 & 34.6 & 9.6 & 33.3 & 18.4 & 43.6 & 39 & 47.4 & 42.5 \\
\hline July & 26.9 & 28 & 27.1 & 27 & 3.5 & 0 & 4.6 & 7.3 & 33.4 & 28.1 & 30.6 & 36.7 \\
\hline August & 28 & 27.6 & 27.4 & 26.8 & 0 & 2.5 & 11.7 & 5.4 & 28 & 26 & 31.1 & 36.8 \\
\hline September & 19.9 & 23.5 & 22.6 & 21.3 & 29.1 & 0 & 11.7 & 16.4 & 40.3 & 26.4 & 37.0 & 42.2 \\
\hline October & 15.2 & 13.4 & 15.9 & 14.2 & 4.4 & 52.8 & 104.5 & 70.3 & 43 & 48.6 & 55.6 & 58.9 \\
\hline November & 6.4 & 7.3 & 7.9 & 6.5 & 53.7 & 99.5 & 83.6 & 91.8 & 47.9 & 68.5 & 72.4 & 64.7 \\
\hline December & -2.2 & 3.7 & 0.75 & 0.2 & 152.6 & 74.6 & 113.6 & 121.8 & 73.4 & 69.8 & 71.6 & 70.7 \\
\hline Total/Ave. & 12.8 & 12.8 & 14.4 & 12.3 & 832.5 & 709.1 & 934.2 & 917.8 & 52.1 & 51.6 & 54.6 & 56.6 \\
\hline
\end{tabular}

are given in table 2 . The majority of the populations yielded higher in terms of green herbage compared to cultivars (Basbag, Bilensoy 80, Savas and Gea). 2016, 2017, 2018 and three years average green herbage yields of average of all genotypes were 51.58, 67.91, 96.39 and $71.96 \mathrm{t} / \mathrm{ha}$, respectively. The highest green herbage average yield (96.39 $\mathrm{t} / \mathrm{ha}$ ) was obtained in the third year of the study. 18 of 27 genotypes were yielded high and positioned in the same statistical group according to three years average green herbage yields. Instead, green herbage yields were highest at Mutluca population in the first year $(65.41 \mathrm{t} / \mathrm{ha})$, at Garip population in the second year $(81.39 \mathrm{t} / \mathrm{ha})$, at Bagliisa population (109.07 t/ha) in the third year, and at Mutluca population for the average of three years $(84.29 \mathrm{t} / \mathrm{ha})$.

2016, 2017, 2018 and three years average hay yields of average of all genotypes were 16.11, $26.31,30.16$ and $24.19 \mathrm{t} / \mathrm{ha}$, respectively. The highest hay yield average $(30.16 \mathrm{t} / \mathrm{ha})$ was obtained in the third year of the study. 14 of 27 genotypes were yielded high and positioned in the same statistical group according to three years average hay yields. Instead, hay yields were highest at Arslanbeyli population in the first year (19.44 t/ha), at Servi population in the second year (30.32 t/ha), at Garip population (39.02 $\mathrm{t} / \mathrm{ha}$ ) in the third year, and at Mutluca population for the average of three years $(27.82 \mathrm{t} / \mathrm{ha})$.

As the year 2016 was the crop establishment year, green herbage and hay yields were lower in this year compared to 2017 and 2018. Under similar arid climatic conditions but in different provinces in Turkey, SEKER (2003) obtained 53.6 t/ ha green herbage and $12.2 \mathrm{t} /$ ha hay yield, TURAN et al. (2017) obtained $37.9 \mathrm{t} / \mathrm{ha}$ green herbage and $13.1 \mathrm{t} / \mathrm{ha}$ hay yield. Under Mediterranean climatic conditions, DEMIROGLU \& AVCIOGLU (2010) obtained 75.4-85.5 t/ha green herbage and 20.5$21.8 \mathrm{t} /$ ha hay yield, GUNDEL et al. (2014) obtained $50.9 \mathrm{t} /$ ha green herbage and $11.3 \mathrm{t} /$ ha hay yield. In a research of JAFARI \& RAZBAN (2018), the forage yield and quality of 49 accessions of alfalfa were determined under dryland farming system during 2005-2007 in the Eastern Azerbaijan. Total forage hay yield were $5.43,4.45 \mathrm{t} / \mathrm{ha}$ in years 2 and 3 , respectively. 5 Genotypes produced average values of $6.5-8.5 \mathrm{t} / \mathrm{ha}$ forage dry matter yield. GOKALP et al. (2017) reported that they obtained 11.8-13.3 t/ha green herbage yield in Tokat, in Turkey.

\section{Crude protein ratios and crude protein yields}

Statsitically significant differences $(\mathrm{P}<0.01)$ were obtained for $\mathrm{CP}$ rates based on genotypes,trial yersand genotype $\mathrm{X}$ year interactions, and for CP yields based on genotypes andtrial yerswhich are are given in table 3 .

2016, 2017, 2018 and three years average CP ratios of average of all genotypes were 24.3, 23.2, 21.6 and $23.0 \%$, respectively. The highest and lowest $\mathrm{CP}$ average rates of $24.3 \%$ and $21.6 \%$ were obtained in the first and third year of the study, respectively. 20 of 27 genotypes were yielded high and positioned in the same statistical group according to three years average $\mathrm{CP}$ ratios. Instead, $\mathrm{CP}$ ratios were highest 
Table 2 - Green herbage and hay yields of alfalfa genotypes.

\begin{tabular}{|c|c|c|c|c|c|c|c|c|c|}
\hline & & \multicolumn{4}{|c|}{ 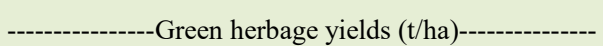 } & \multicolumn{4}{|c|}{ 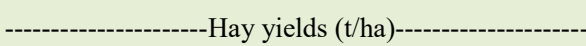 } \\
\hline & Genotypes & 2016 & 2017 & 2018 & Mean & 2016 & 2017 & 2018 & Mean $^{* *}$ \\
\hline 1 & Garip & 54.14 & 81.39 & 103.71 & $79.75 \mathrm{ab}$ & 17.74 & 26.70 & 39.02 & $27.82 \mathrm{ab}$ \\
\hline 2 & Küçüktekören & 46.49 & 64.89 & 90.18 & $67.19 \mathrm{bc}$ & 14.36 & 25.19 & 27.65 & $22.40 \mathrm{~cd}$ \\
\hline 3 & Kurudere & 51.34 & 67.31 & 80.65 & $66.43 \mathrm{bc}$ & 16.29 & 22.55 & 30.19 & $23.01 \mathrm{bcd}$ \\
\hline 4 & Kumgeçit & 44.76 & 64.94 & 95.20 & $68.30 \mathrm{abc}$ & 14.62 & 27.37 & 26.80 & $22.93 \mathrm{bcd}$ \\
\hline 5 & Kültür Mah. & 43.74 & 66.62 & 90.34 & $66.90 \mathrm{bc}$ & 14.31 & 23.29 & 27.21 & $21.60 \mathrm{~d}$ \\
\hline 6 & Ortaköy & 46.02 & 59.75 & 79.31 & $61.69 \mathrm{c}$ & 14.30 & 22.28 & 27.29 & $21.29 \mathrm{~d}$ \\
\hline 7 & Sarıçiçek-1 & 47.88 & 76.08 & 10.420 & $76.05 \mathrm{abc}$ & 15.04 & 29.94 & 34.04 & $26.34 \mathrm{a}-\mathrm{d}$ \\
\hline 8 & Sarıçiçek-2 & 42.69 & 62.37 & 94.72 & $66.60 \mathrm{bc}$ & 13.55 & 25.93 & 27.67 & $22.38 \mathrm{~cd}$ \\
\hline 9 & Sarıçiçek-3 & 48.72 & 67.63 & 99.80 & $72.05 \mathrm{abc}$ & 14.85 & 29.63 & 29.94 & $24.81 \mathrm{a}-\mathrm{d}$ \\
\hline 10 & Yelesen & 43.74 & 56.28 & 85.65 & $61.89 \mathrm{c}$ & 13.93 & 26.05 & 27.31 & $22.43 \mathrm{~cd}$ \\
\hline 11 & Çeltiksuyu-1 & 46.42 & 62.19 & 100.02 & $69.54 \mathrm{abc}$ & 14.74 & 26.28 & 28.95 & $23.32 \mathrm{bcd}$ \\
\hline 12 & Çeltiksuyu-2 & 50.29 & 63.81 & 89.01 & $67.70 \mathrm{bc}$ & 15.73 & 23.84 & 30.41 & $23.33 \mathrm{bcd}$ \\
\hline 13 & Çeltiksuyu-3 & 50.75 & 73.93 & 93.12 & $72.60 \mathrm{abc}$ & 16.70 & 25.74 & 33.18 & $25.21 \mathrm{a}-\mathrm{d}$ \\
\hline 14 & Meşedal1-1 & 57.45 & 75.05 & 100.27 & $77.59 \mathrm{abc}$ & 17.20 & 26.85 & 32.39 & $25.48 \mathrm{a}-\mathrm{d}$ \\
\hline 15 & Meşedalı-2 & 52.83 & 69.07 & 96.06 & $72.65 \mathrm{abc}$ & 15.52 & 27.74 & 30.95 & 24.74 a-d \\
\hline 16 & Servi & 58.26 & 78.11 & 108.68 & $81.68 \mathrm{ab}$ & 18.68 & 30.32 & 36.59 & $28.53 \mathrm{a}$ \\
\hline 17 & Çevirme & 45.96 & 64.87 & 92.99 & $67.94 \mathrm{bc}$ & 14.83 & 25.64 & 28.90 & $23.12 \mathrm{bcd}$ \\
\hline 18 & Arslanbeyli & 62.17 & 66.68 & 98.19 & $75.68 \mathrm{abc}$ & 19.44 & 25.35 & 29.64 & $24.81 \mathrm{a}-\mathrm{d}$ \\
\hline 19 & Mutluca & 65.41 & 80.08 & 107.38 & $84.29 \mathrm{a}$ & 19.18 & 28.43 & 34.33 & $27.31 \mathrm{abc}$ \\
\hline 20 & Bağliisa & 56.70 & 73.43 & 109.07 & $79.73 \mathrm{ab}$ & 18.06 & 29.01 & 31.33 & $26.13 \mathrm{a}-\mathrm{d}$ \\
\hline 21 & Taşlıçay & 46.54 & 64.23 & 94.03 & $68.26 \mathrm{abc}$ & 14.93 & 25.69 & 28.77 & $23.13 \mathrm{bcd}$ \\
\hline 22 & Şenköy-1 & 53.44 & 63.65 & 106.83 & $74.64 \mathrm{abc}$ & 17.39 & 28.89 & 28.38 & $24.88 \mathrm{a}-\mathrm{d}$ \\
\hline 23 & Şenköy-2 & 49.49 & 66.81 & 98.85 & $71.72 a b c$ & 15.00 & 25.48 & 29.32 & $23.27 \mathrm{bcd}$ \\
\hline 24 & Başbağ & 60.78 & 67.75 & 95.74 & $74.76 \mathrm{abc}$ & 18.12 & 25.51 & 29.51 & $24.38 \mathrm{a}-\mathrm{d}$ \\
\hline 25 & Bilensoy 80 & 60.10 & 64.63 & 93.24 & $72.65 \mathrm{abc}$ & 18.15 & 24.65 & 27.60 & 23.47 a-d \\
\hline 26 & Gea & 57.58 & 73.02 & 99.94 & $76.85 \mathrm{abc}$ & 17.98 & 26.83 & 32.01 & $25.61 \mathrm{a}-\mathrm{d}$ \\
\hline \multirow[t]{4}{*}{27} & Savaş & 48.95 & 59.05 & 95.36 & $67.79 \mathrm{bc}$ & 14.20 & 25.16 & 24.92 & $21.43 \mathrm{~d}$ \\
\hline & Mean & $51.58 \mathrm{C}$ & $67.91 \mathrm{~B}$ & $96.39 \mathrm{~A}$ & 71.96 & $16.11 \mathrm{C}$ & $26.31 \mathrm{~B}$ & $30.16 \mathrm{~A}$ & 24.19 \\
\hline & LSD (0.05) & \multicolumn{4}{|c|}{ Year $(\mathrm{Y}): 34.05^{* *}, \begin{array}{c}\text { Genotype }(\mathrm{G}): 16.25^{* *}, \mathrm{Y} \text { x G }: \text { not } \\
\text { significant }\end{array}$} & \multicolumn{4}{|c|}{ 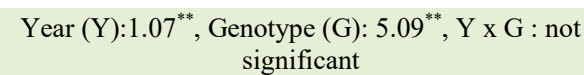 } \\
\hline & & \multicolumn{4}{|c|}{ CV: $\% 12.73,{ }^{* *} \mathrm{P} \leq 0.01$} & \multicolumn{4}{|c|}{$\mathrm{CV}: \% 11.87,{ }^{* *} \mathrm{P} \leq 0.01$} \\
\hline
\end{tabular}

at Bilensoy 80 variety in the first year (25.7\%), at Taslicay and Senkoy-1 population in the second year (both $24.8 \%$ ), at Kumgecit population $(24.0 \%$ ) in the third year, and at Kumgecit population for the average of three years $(24.2 \%)$. Under similar arid climatic conditions in Turkey, ENGIN \& MUT (2017) and CACAN et al. (2018) was obtained $25.0 \%$ and 23.9 $25.9 \%$ respectively. BASBAG et al. (2009), collected 11 alfalfa clones from Southeastern and Eastern Regions of Turkey and determined a significant variation in quality properties of alfalfa clones for crude protein (17.3-23.2\%). In a study of Basbag et al., (2017), 16 individual plants were selected from the Southeastern Turkey from their natural habitat and clonally propagated in field trials where crude protein content ranged between 17.33 to $24.60 \%$.
2016, 2017, 2018 and three years average CP yields of average of all genotypes were 3.74, $6.39,6.50$ and $5.54 \mathrm{t} / \mathrm{ha}$, respectively. The highest and lowest $\mathrm{CP}$ yield averages were obtained in the third and first years of the study, respectively. 23 of 27 genotypes were yielded high and positioned in the same statistical group according to three years average $\mathrm{CP}$ yields. Instead, CP yields were highest at Arslanbeyli and Bagliisa populations in the first year (both $4.42 \mathrm{t} / \mathrm{ha}$ ), at Saricicek population in the second year (7.24 t/ha), at Servi population $(8.07 \mathrm{t} /$ ha) in the third year, and at Servi population for the average of three years $(6.40 \mathrm{t} / \mathrm{ha})$. Under similar arid climatic conditions in Turkey, SENGUL et al. (2003) was obtained 2.46-3.21 t/ha, TURAN et al. (2017) was obtained 1.35-2.83 t/ha and ENGIN \& 
Table 3 - Crude protein ratios and crude protein yields of alfalfa genotypes.

\begin{tabular}{|c|c|c|c|c|c|c|c|c|c|}
\hline & & \multicolumn{4}{|c|}{------------------Crude protein ratios (\%)---------------- } & \multicolumn{4}{|c|}{ 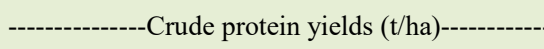 } \\
\hline & Genotypes & 2016 & 2017 & 2018 & Mean & 2016 & 2017 & 2018 & Mean \\
\hline 1 & Garip & 23.4 a-o & $22.5 \mathrm{a}-\mathrm{p}$ & 19.2 qrs & $21.7 \mathrm{e}$ & 4.00 & 6.26 & 7.53 & $5.93 \mathrm{ab}$ \\
\hline 2 & Küçüktekören & $24.9 \mathrm{a}-\mathrm{i}$ & $23.7 \mathrm{a}-\mathrm{n}$ & $23.4 \mathrm{a}-\mathrm{o}$ & $24.0 \mathrm{ab}$ & 3.40 & 6.27 & 6.48 & $5.39 \mathrm{ab}$ \\
\hline 3 & Kurudere & $23.7 \mathrm{a}-\mathrm{n}$ & $23.3 \mathrm{a}-\mathrm{p}$ & $21.2 \mathrm{k}-\mathrm{s}$ & $22.8 \mathrm{a}-\mathrm{e}$ & 3.82 & 5.35 & 6.42 & $5.20 \mathrm{ab}$ \\
\hline 4 & Kumgeçit & $25.5 \mathrm{ab}$ & $23.2 \mathrm{a}-\mathrm{p}$ & $24.0 \mathrm{a}-\mathrm{m}$ & $24.2 \mathrm{a}$ & 3.42 & 6.99 & 6.44 & $5.62 \mathrm{ab}$ \\
\hline 5 & Kültür Mah. & $25.2 \mathrm{a}-\mathrm{f}$ & 24.4 a-k & $20.2 \mathrm{p}-\mathrm{s}$ & $23.3 \mathrm{a}-\mathrm{e}$ & 3.51 & 5.88 & 5.50 & $4.96 \mathrm{~b}$ \\
\hline 6 & Ortaköy & $25.2 \mathrm{a}-\mathrm{f}$ & $22.1 \mathrm{c}-\mathrm{r}$ & $22.1 \mathrm{e}-\mathrm{r}$ & $23.2 \mathrm{a}-\mathrm{e}$ & 3.17 & 5.63 & 6.03 & $4.94 \mathrm{~b}$ \\
\hline 7 & Sarıçiçek-1 & 23.8 a-n & $21.8 \mathrm{~h}-\mathrm{r}$ & $21.7 \mathrm{i}-\mathrm{s}$ & $22.4 \mathrm{~b}-\mathrm{e}$ & 3.28 & 7.12 & 7.39 & $5.93 \mathrm{ab}$ \\
\hline 8 & Sarıçiçek-2 & $23.1 \mathrm{a}-\mathrm{p}$ & 22.5 a-p & $21.11-\mathrm{s}$ & $22.2 \mathrm{de}$ & 3.06 & 5.98 & 5.81 & $4.95 \mathrm{~b}$ \\
\hline 9 & Sarıçiçek-3 & $24.4 \mathrm{a}-\mathrm{k}$ & 23.7 a-n & $20.6 \mathrm{n}-\mathrm{s}$ & 22.9 a-e & 3.51 & 7.24 & 6.16 & $5.64 \mathrm{ab}$ \\
\hline 10 & Yelesen & $24.3 \mathrm{a}-1$ & $22.4 \mathrm{~b}-\mathrm{q}$ & $22.5 \mathrm{a}-\mathrm{p}$ & 23.1 a-e & 3.19 & 6.34 & 6.14 & $5.22 \mathrm{ab}$ \\
\hline 11 & Çeltiksuyu-1 & $24.3 \mathrm{a}-\mathrm{m}$ & $23.7 \mathrm{a}-\mathrm{n}$ & 20.3 o-s & 22.8 a-e & 3.49 & 6.37 & 5.90 & $5.26 \mathrm{ab}$ \\
\hline 12 & Çeltiksuyu-2 & $25.4 \mathrm{abc}$ & 23.0 a-p & $21.1 \mathrm{~m}-\mathrm{s}$ & $23.1 \mathrm{a}-\mathrm{e}$ & 3.62 & 6.03 & 6.41 & $5.35 \mathrm{ab}$ \\
\hline 13 & Çeltiksuyu-3 & 22.9 a-p & $23.1 \mathrm{a}-\mathrm{p}$ & $21.0 \mathrm{~m}-\mathrm{s}$ & 22.4 cde & 3.86 & 5.92 & 6.99 & $5.59 \mathrm{ab}$ \\
\hline 14 & Meşedalı-1 & $23.8 \mathrm{a}-\mathrm{m}$ & $23.2 \mathrm{a}-\mathrm{p}$ & 21.8 h-r & 22.9 a-e & 3.96 & 6.38 & 7.06 & $5.80 \mathrm{ab}$ \\
\hline 15 & Meşedal1-2 & 23.0 a-p & $22.4 b-q$ & 22.6 a-p & 22.7 a-e & 3.48 & 6.40 & 6.98 & $5.62 \mathrm{ab}$ \\
\hline 16 & Servi & 23.4 a-p & $21.7 \mathrm{i}-\mathrm{s}$ & $22.2 \mathrm{c}-\mathrm{r}$ & $22.4 \mathrm{~b}-\mathrm{e}$ & 4.05 & 7.08 & 8.07 & $6.40 \mathrm{a}$ \\
\hline 17 & Çevirme & 22.9 a-p & 22.9 a-p & $22.1 \mathrm{~d}-\mathrm{r}$ & 22.6 a-e & 3.40 & 5.87 & 6.39 & $5.22 \mathrm{ab}$ \\
\hline 18 & Arslanbeyli & $23.1 \mathrm{a}-\mathrm{p}$ & 22.8 a-p & 23.3 a-p & $23.1 \mathrm{a}-\mathrm{e}$ & 4.42 & 5.83 & 6.92 & $5.72 \mathrm{ab}$ \\
\hline 19 & Mutluca & $25.0 \mathrm{a}-\mathrm{h}$ & 22.7 a-p & $19.0 \mathrm{rs}$ & $22.2 \mathrm{de}$ & 4.35 & 7.09 & 6.50 & $5.98 \mathrm{ab}$ \\
\hline 20 & Bağliisa & $24.2 \mathrm{a}-\mathrm{m}$ & $24.4 \mathrm{a}-\mathrm{k}$ & $18.5 \mathrm{~s}$ & 22.4 b-e & 4.42 & 7.04 & 5.80 & $5.75 \mathrm{ab}$ \\
\hline 21 & Taşlıçay & $25.2 \mathrm{a}-\mathrm{g}$ & $24.8 \mathrm{a}-\mathrm{i}$ & $21.5 \mathrm{j}-\mathrm{s}$ & $23.8 \mathrm{a}-\mathrm{d}$ & 3.69 & 6.47 & 6.18 & $5.45 \mathrm{ab}$ \\
\hline 22 & Şenköy-1 & $23.9 \mathrm{a}-\mathrm{m}$ & $24.8 \mathrm{a}-\mathrm{i}$ & 23.4 a-p & $24.0 \mathrm{ab}$ & 4.31 & 6.89 & 6.63 & $5.94 \mathrm{ab}$ \\
\hline 23 & Şenköy-2 & $25.4 \mathrm{a}-\mathrm{d}$ & 22.7 a-p & 22.7 a-p & $23.6 \mathrm{a}-\mathrm{d}$ & 3.41 & 6.44 & 6.66 & $5.50 \mathrm{ab}$ \\
\hline 24 & Başbağ & $24.8 \mathrm{a}-\mathrm{i}$ & 23.6 a-n & $21.4 \mathrm{j}-\mathrm{s}$ & $23.3 \mathrm{a}-\mathrm{e}$ & 4.29 & 6.32 & 6.30 & $5.63 \mathrm{ab}$ \\
\hline 25 & Bilensoy 80 & $25.7 \mathrm{a}$ & 23.4 a-p & $22.5 \mathrm{a}-\mathrm{p}$ & $23.9 \mathrm{abc}$ & 4.25 & 6.35 & 6.22 & $5.61 \mathrm{ab}$ \\
\hline 26 & Gea & 25.3 a-e & $23.5 \mathrm{a}-\mathrm{o}$ & $22.0 \mathrm{~g}-\mathrm{r}$ & 23.6 a-e & 4.23 & 6.78 & 7.04 & $6.01 \mathrm{ab}$ \\
\hline \multirow[t]{4}{*}{27} & Savaş & $24.6 \mathrm{a}-\mathrm{j}$ & $23.1 \mathrm{a}-\mathrm{p}$ & $22.0 \mathrm{f}-\mathrm{r}$ & $23.3 \mathrm{a}-\mathrm{e}$ & 3.29 & 6.20 & 5.49 & $4.99 \mathrm{~b}$ \\
\hline & Mean & $24.3 \mathrm{~A}$ & $23.2 \mathrm{~B}$ & $21.6 \mathrm{C}$ & 23.0 & $3.74 \mathrm{~B}$ & $6.39 \mathrm{~A}$ & $6.50 \mathrm{~A}$ & 5.54 \\
\hline & $\operatorname{LSD}(0.05)$ & \multicolumn{4}{|c|}{ Year $(Y): 0.34^{* *}$, Genotype $(\mathrm{G}): 1.64^{* *}$, Y x G : $3.24^{* *}$} & \multicolumn{4}{|c|}{$\begin{array}{c}\text { Year (Y): } 0.25^{* *}, \text { Genotype }(\mathrm{G}): 1.22^{* *}, \mathrm{Y} \text { x G } \\
\text { not significant }\end{array}$} \\
\hline & & \multicolumn{4}{|c|}{$\mathrm{CV}(\%): 4.00,{ }^{* *} \mathrm{P} \leq 0.01$} & \multicolumn{4}{|c|}{ CV:\%12.36, ${ }^{* *} \mathrm{P} \leq 0.01$} \\
\hline
\end{tabular}

MUT (2017) 4.29 t/ha CP yields. These CP rate and yield values are in accordance with our results.

\section{$A D F$ and NDF ratios}

Statistically significant differences $(\mathrm{P}<0.01)$ were obtained for ADF based on genotypes, trial yers and genotype $\mathrm{x}$ year interactions, and for NDF rates based on genotypes and genotype $x$ year interactions which are given in table 4 .

2016, 2017, 2018 and three years average ADF ratios of average of all genotypes were 21.8, 23.6, 23.6 and $23.0 \%$, respectively. ADF ratio averages were same and high for second and third year compared to first year of trials. 21 of 27 genotypes were yielded high and positioned in the same statistical group according to three years average $\mathrm{ADF}$ ratios. ADF ratios were lowest(indicator of high quality) at Saricicek-3 population in the first year $(18.7 \%)$, at Gea variety in the second year $(18.7 \%)$, at Kumgecit population $(20.7 \%)$ in the third year, and at Kumgecit population for the average of three years (20.8\%).

2016, 2017, 2018 and three years average NDF ratios of average of all genotypes were 39.0, 37.9, 38.8 and $38.6 \%$, respectively. 14 of 27 genotypes were ranked low(indicator of high quality) and positioned in the same statistical group according to three years average NDF ratios. NDF ratios were lowest at Bagliisa population in the first year (35.9\%), at Bilensoy 80 and Geavarieties in the second year (both $27.5 \%$ ), at Savas variety $(34.8 \%)$ in the third year, and at Gea varity for the average of three years (33.3\%). KARAKOY \& SARAC (2018) reported ADF rate 
Table 4 - Acid detergent fiber (ADF) and neutral detergent fiber (NDF) ratios of alfalfa genotypes.

\begin{tabular}{|c|c|c|c|c|c|c|c|c|c|}
\hline & & \multicolumn{4}{|c|}{ 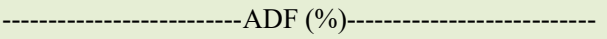 } & \multicolumn{4}{|c|}{ - } \\
\hline & Genotypes & 2016 & 2017 & 2018 & Mean & 2016 & 2017 & 2018 & Mean \\
\hline 1 & Garip & $22.4 \mathrm{~b}-\mathrm{m}$ & $24.7 \mathrm{a}-\mathrm{j}$ & $25.5 \mathrm{a}-\mathrm{f}$ & $24.2 \mathrm{abc}$ & 39.0 a-e & $41.5 \mathrm{a}-\mathrm{d}$ & $41.3 \mathrm{a}-\mathrm{d}$ & $40.6 \mathrm{ab}$ \\
\hline 2 & Küçüktekören & $23.1 \mathrm{a}-\mathrm{m}$ & 25.8 a-e & 23.8 a-1 & $24.2 \mathrm{ab}$ & 40.8 a-e & $44.4 \mathrm{abc}$ & $38.0 \mathrm{a}-\mathrm{f}$ & $41.1 \mathrm{a}$ \\
\hline 3 & Kurudere & $25.2 \mathrm{a}-\mathrm{g}$ & 23.9 a-1 & $25.1 \mathrm{a}-\mathrm{h}$ & $24.7 \mathrm{a}$ & $43.8 \mathrm{abc}$ & 40.2 a-e & 37.9 a-f & $40.6 \mathrm{ab}$ \\
\hline 4 & Kumgeçit & 20.1 h-m & $21.6 \mathrm{c}-\mathrm{m}$ & $20.7 \mathrm{f}-\mathrm{m}$ & $20.8 \mathrm{f}$ & 36.7 a-f & $33.8 \mathrm{c}-\mathrm{f}$ & $34.3 \mathrm{~b}-\mathrm{f}$ & $34.9 \mathrm{bcd}$ \\
\hline 5 & Kültür Mah. & 20.2 h-m & $23.6 \mathrm{a}-\mathrm{m}$ & $25.0 \mathrm{a}-\mathrm{h}$ & $22.9 \mathrm{a}-\mathrm{f}$ & $36.2 \mathrm{a}-\mathrm{f}$ & $37.4 \mathrm{a}-\mathrm{f}$ & 40.7 a-e & $38.1 \mathrm{a}-\mathrm{d}$ \\
\hline 6 & Ortaköy & $24.7 \mathrm{a}-\mathrm{i}$ & $23.2 \mathrm{a}-\mathrm{m}$ & $22.2 \mathrm{~b}-\mathrm{m}$ & $23.4 \mathrm{a}-\mathrm{e}$ & $42.9 \mathrm{abc}$ & $37.8 \mathrm{a}-\mathrm{f}$ & 39.6 a-e & $40.1 \mathrm{abc}$ \\
\hline 7 & Sarıçiçek-1 & $24.3 \mathrm{a}-\mathrm{k}$ & $23.5 \mathrm{a}-\mathrm{m}$ & $22.1 \mathrm{c}-\mathrm{m}$ & $23.3 \mathrm{a}-\mathrm{f}$ & $46.1 \mathrm{a}$ & 36.9 a-f & $35.7 \mathrm{a}-\mathrm{f}$ & $39.6 \mathrm{abc}$ \\
\hline 8 & Sarıçiçek-2 & 23.6 a-m & $23.2 \mathrm{a}-\mathrm{m}$ & 22.9 a-m & $23.2 \mathrm{a}-\mathrm{f}$ & 40.6 a-e & $41.1 \mathrm{a}-\mathrm{e}$ & 39.7 a-e & $40.5 \mathrm{ab}$ \\
\hline 9 & Sarıçiçek-3 & $18.7 \mathrm{~m}$ & 23.4 a-m & $21.6 \mathrm{c}-\mathrm{m}$ & $21.3 \mathrm{def}$ & $37.3 \mathrm{a}-\mathrm{f}$ & $38.0 \mathrm{a}-\mathrm{f}$ & $37.2 \mathrm{a}-\mathrm{f}$ & $37.5 \mathrm{a}-\mathrm{d}$ \\
\hline 10 & Yelesen & $22.2 \mathrm{~b}-\mathrm{m}$ & $23.3 \mathrm{a}-\mathrm{m}$ & $22.9 \mathrm{a}-\mathrm{m}$ & $22.8 \mathrm{a}-\mathrm{f}$ & 39.3 a-e & 40.0 a-e & $37.1 \mathrm{a}-\mathrm{f}$ & 38.8 a-d \\
\hline 11 & Çeltiksuyu-1 & $22.4 \mathrm{~b}-\mathrm{m}$ & $25.2 \mathrm{a}-\mathrm{g}$ & $23.4 \mathrm{a}-\mathrm{m}$ & 23.7 a-d & 40.2 a-e & 36.9 a-f & $37.3 \mathrm{a}-\mathrm{f}$ & $38.1 \mathrm{a}-\mathrm{d}$ \\
\hline 12 & Çeltiksuyu-2 & $22.9 \mathrm{a}-\mathrm{m}$ & $21.9 \mathrm{c}-\mathrm{m}$ & $21.7 \mathrm{c}-\mathrm{m}$ & $22.2 \mathrm{~b}-\mathrm{f}$ & 39.9 a-e & $34.2 \mathrm{~b}-\mathrm{f}$ & $37.1 \mathrm{a}-\mathrm{f}$ & $37.1 \mathrm{a}-\mathrm{d}$ \\
\hline 13 & Çeltiksuyu-3 & $20.7 \mathrm{f}-\mathrm{m}$ & $27.2 \mathrm{ab}$ & $24.2 \mathrm{a}-\mathrm{k}$ & $24.0 \mathrm{abc}$ & $37.2 \mathrm{a}-\mathrm{f}$ & $40.1 \mathrm{a}-\mathrm{e}$ & $45.6 \mathrm{ab}$ & $41.0 \mathrm{a}$ \\
\hline 14 & Meşedal1-1 & $21.1 \mathrm{~d}-\mathrm{m}$ & $24.8 \mathrm{a}-\mathrm{i}$ & $23.4 \mathrm{a}-\mathrm{m}$ & $23.1 \mathrm{a}-\mathrm{f}$ & $38.2 \mathrm{a}-\mathrm{f}$ & $41.2 \mathrm{a}-\mathrm{d}$ & $36.8 \mathrm{a}-\mathrm{f}$ & $38.7 \mathrm{a}-\mathrm{d}$ \\
\hline 15 & Meşedal1-2 & 20.8 e-m & $23.6 \mathrm{a}-\mathrm{m}$ & $25.0 \mathrm{a}-\mathrm{h}$ & $23.2 \mathrm{a}-\mathrm{f}$ & $37.6 \mathrm{a}-\mathrm{f}$ & $42.1 \mathrm{a}-\mathrm{d}$ & 40.2 a-e & $40.0 \mathrm{abc}$ \\
\hline 16 & Servi & 24.0 a-1 & $24.4 \mathrm{a}-\mathrm{k}$ & 25.8 a-e & $24.7 \mathrm{a}$ & 40.8 a-e & $41.0 \mathrm{a}-\mathrm{d}$ & 38.8 a-f & $40.2 \mathrm{ab}$ \\
\hline 17 & Çevirme & $22.6 \mathrm{a}-\mathrm{m}$ & $23.4 \mathrm{a}-\mathrm{m}$ & $22.8 \mathrm{a}-\mathrm{m}$ & $22.9 \mathrm{a}-\mathrm{f}$ & 40.2 a-e & $41.2 \mathrm{a}-\mathrm{d}$ & $41.9 \mathrm{a}-\mathrm{d}$ & $41.1 \mathrm{a}$ \\
\hline 18 & Arslanbeyli & 19.8 i-m & $25.4 \mathrm{a}-\mathrm{f}$ & 24.0 a-1 & $23.1 \mathrm{a}-\mathrm{f}$ & $36.2 \mathrm{a}-\mathrm{f}$ & 40.3 a-e & $37.3 \mathrm{a}-\mathrm{f}$ & $37.9 \mathrm{a}-\mathrm{d}$ \\
\hline 19 & Mutluca & 20.2 g-m & $25.1 \mathrm{a}-\mathrm{h}$ & $26.4 \mathrm{abc}$ & $23.9 \mathrm{abc}$ & $37.2 \mathrm{a}-\mathrm{f}$ & 37.8 a-f & $43.7 \mathrm{abc}$ & $39.6 \mathrm{abc}$ \\
\hline 20 & Bağliiisa & $19.7 \mathrm{j}-\mathrm{m}$ & $26.1 \mathrm{a}-\mathrm{d}$ & $26.4 \mathrm{abc}$ & $24.1 \mathrm{abc}$ & 35.9 a-f & $41.3 \mathrm{a}-\mathrm{e}$ & $45.9 \mathrm{a}$ & $41.0 \mathrm{a}$ \\
\hline 21 & Taşlıçay & $19.7 \mathrm{klm}$ & $23.2 \mathrm{a}-\mathrm{m}$ & $24.3 \mathrm{a}-\mathrm{k}$ & $22.4 \mathrm{a}-\mathrm{f}$ & 36.9 a-f & 39.0 a-e & 39.4 a-e & $38.4 \mathrm{a}-\mathrm{d}$ \\
\hline 22 & Şenköy-1 & $21.0 \mathrm{e}-\mathrm{m}$ & $27.5 \mathrm{a}$ & $24.4 \mathrm{a}-\mathrm{k}$ & $24.3 \mathrm{ab}$ & $38.0 \mathrm{a}-\mathrm{f}$ & $43.0 \mathrm{abc}$ & $38.5 \mathrm{a}-\mathrm{f}$ & $39.9 \mathrm{abc}$ \\
\hline 23 & Şenköy-2 & $21.6 \mathrm{c}-\mathrm{m}$ & $24.7 \mathrm{a}-\mathrm{i}$ & $21.8 \mathrm{c}-\mathrm{m}$ & $22.7 \mathrm{a}-\mathrm{f}$ & $38.1 \mathrm{a}-\mathrm{f}$ & $38.2 \mathrm{a}-\mathrm{f}$ & $35.3 \mathrm{a}-\mathrm{f}$ & $37.2 \mathrm{a}-\mathrm{d}$ \\
\hline 24 & Başbağ & $21.8 \mathrm{c}-\mathrm{m}$ & $20.3 \mathrm{~g}-\mathrm{h}$ & $23.0 \mathrm{a}-\mathrm{m}$ & $21.7 \mathrm{c}-\mathrm{f}$ & $38.1 \mathrm{a}-\mathrm{f}$ & $29.4 \mathrm{ef}$ & $40.2 \mathrm{a}-\mathrm{e}$ & $35.9 \mathrm{a}-\mathrm{d}$ \\
\hline 25 & Bilensoy 80 & $20.6 \mathrm{f}-\mathrm{m}$ & $19.2 \mathrm{~lm}$ & $23.0 \mathrm{a}-\mathrm{m}$ & 20.9 ef & $37.7 \mathrm{a}-\mathrm{f}$ & $27.5 \mathrm{f}$ & 38.0 a-f & $34.4 \mathrm{~cd}$ \\
\hline 26 & Gea & $20.7 \mathrm{f}-\mathrm{m}$ & $18.7 \mathrm{~m}$ & $23.2 \mathrm{a}-\mathrm{m}$ & 20.9 ef & $36.8 \mathrm{a}-\mathrm{f}$ & $27.5 \mathrm{f}$ & 35.7 a-f & $33.3 \mathrm{~d}$ \\
\hline \multirow[t]{4}{*}{27} & Savaş & $24.6 \mathrm{a}-\mathrm{k}$ & $21.1 \mathrm{~d}-\mathrm{m}$ & $22.6 \mathrm{a}-\mathrm{m}$ & $22.8 \mathrm{a}-\mathrm{f}$ & $41.7 \mathrm{a}-\mathrm{e}$ & $31.4 \mathrm{def}$ & $34.8 \mathrm{a}-\mathrm{f}$ & $36.0 \mathrm{a}-\mathrm{d}$ \\
\hline & Mean & $21.8 \mathrm{~B}$ & $23.6 \mathrm{~A}$ & $23.6 \mathrm{~A}$ & 23.0 & 39.0 & 37.9 & 38.8 & 38.6 \\
\hline & LSD (0.05) & \multicolumn{4}{|c|}{ Year (Y):0.53 ${ }^{* *}$, Genotype (G): $2.53^{* *}$, Y x G : $5.01^{* *}$} & \multicolumn{4}{|c|}{$\begin{array}{l}\text { Year (Y): not significant, Genotype }(\mathrm{G}): 5.76^{* *} \text {, Y x } \\
\text { G : } 11.42^{* *}\end{array}$} \\
\hline & & \multicolumn{4}{|c|}{$\mathrm{CV}(\%): 6.20,{ }^{* *} \mathrm{P} \leq 0.01$} & \multicolumn{4}{|c|}{ CV:\%8.42, ${ }^{* *} \mathrm{P} \leq 0.01$} \\
\hline
\end{tabular}

between 42.66-44.19\% and NDF rate between 51.38$53.78 \%$ in Sivas province of Turkey,in accordance with our study. BASBAG et al. (2009), collected 11 alfalfa clones from Southeastern and Eastern Regions of Turkey and determined a significant variation in quality properties of alfalfa clones for ADF (16.8$33.3 \%$ ) and NDF (20.3-35.2\%). In a study of Basbag et al., (2017), 16 individual plants were selected from the Southeastern Turkey from their natural habitat and clonally propagated in field trials where ADF values ranged from 20.52 to $37.71 \%$ and NDF values were between 22.16 and $41.22 \%$.

\section{$D D M$ and $R F V$ values}

It was found that there were statistically significant $(\mathrm{P}<0.01)$ differences among genotypes and average of years in terms of digestible dry matter ratios and relative feed values, but there was no statistically significant difference among years in terms of relative feed value. DDM and RFV values of alfalfa genotypes examined in the study are given in table 5 .

2016, 2017, 2018 and three years average DDM of average of all genotypes were 71.9, 70.5, 70.5 and $71.0 \%$, respectively. DDM averages were higherin the first year compared to second and third year of trials. 17 of 27 genotypes were yielded high and positioned in the same statistical group according to three years average DDM values. DDM values were highest at Saricicek-3 population in the first year $(74.3 \%)$, at Gea variety in the second year (74.3\%), at Kumgecit population (72.8\%) in the third

Ciência Rural, v.50, n.11, 2020. 
Table 5 - Digestible dry matter (DDM, \%) and reletive feed value (RFV)of alfalfa genotypes.

\begin{tabular}{|c|c|c|c|c|c|c|c|c|c|}
\hline & & \multicolumn{4}{|c|}{ 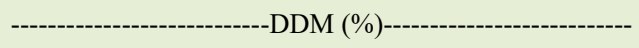 } & \multicolumn{4}{|c|}{--------------------------------'RFV---------------------------- } \\
\hline & Populations & 2016 & 2017 & 2018 & Mean & 2016 & 2017 & 2018 & Mean \\
\hline 1 & Garip & $71.5 \mathrm{a}-1$ & $69.7 \mathrm{~d}-\mathrm{m}$ & 69.0 h-m & 70.1 def & $172.1 \mathrm{cde}$ & $156.1 \mathrm{de}$ & $157.1 \mathrm{cde}$ & $161.8 \mathrm{e}$ \\
\hline 2 & Küçüktekören & 70.9 a-m & $68.8 \mathrm{i}-\mathrm{m}$ & $70.3 \mathrm{~b}-\mathrm{m}$ & $70.0 \mathrm{ef}$ & 161.9 cde & $144.1 \mathrm{de}$ & 172.6 cde & $159.6 \mathrm{e}$ \\
\hline 3 & Kurudere & $69.3 \mathrm{~g}-\mathrm{m}$ & $70.3 \mathrm{~b}-\mathrm{m}$ & $69.4 \mathrm{f}-\mathrm{m}$ & $69.6 \mathrm{f}$ & $147.8 \mathrm{de}$ & $164.0 \mathrm{cde}$ & 170.4 cde & $160.8 \mathrm{e}$ \\
\hline 4 & Kumgeçit & $73.2 \mathrm{a}-\mathrm{f}$ & $72.1 \mathrm{a}-\mathrm{k}$ & $72.8 \mathrm{a}-\mathrm{h}$ & $72.7 \mathrm{a}$ & $186.8 \mathrm{~b}-\mathrm{e}$ & 199.0 a-e & 199.4 a-d & $195.1 \mathrm{abc}$ \\
\hline 5 & Kültür Mah. & $73.2 \mathrm{a}-\mathrm{f}$ & $70.5 \mathrm{a}-\mathrm{m}$ & $69.5 \mathrm{f}-\mathrm{m}$ & $71.0 \mathrm{a}-\mathrm{f}$ & $188.4 \mathrm{~b}-\mathrm{e}$ & $176.2 \mathrm{~b}-\mathrm{e}$ & 159.9 cde & $174.8 \mathrm{~b}-\mathrm{e}$ \\
\hline 6 & Ortaköy & $69.6 \mathrm{e}-\mathrm{m}$ & $70.8 \mathrm{a}-\mathrm{m}$ & $71.6 \mathrm{a}-1$ & $70.7 \mathrm{~b}-\mathrm{f}$ & $152.3 \mathrm{de}$ & $175.2 \mathrm{~b}-\mathrm{e}$ & $170.6 \mathrm{cde}$ & $166.0 \mathrm{cde}$ \\
\hline 7 & Sarıçiçek-1 & $70.0 \mathrm{c}-\mathrm{m}$ & 70.6 a-m & $71.7 \mathrm{a}-\mathrm{k}$ & 70.8 a-f & $141.3 \mathrm{de}$ & $178.5 \mathrm{~b}-\mathrm{e}$ & $187.3 \mathrm{~b}-\mathrm{e}$ & $169.0 \mathrm{cde}$ \\
\hline 8 & Sarıçiçek-2 & $70.5 \mathrm{a}-\mathrm{m}$ & 70.9 a-m & $71.0 \mathrm{a}-\mathrm{m}$ & 70.8 a-f & $162.2 \mathrm{cde}$ & $161.9 \mathrm{cde}$ & 167.8 cde & $164.0 \mathrm{de}$ \\
\hline 9 & Sarıçiçek-3 & $74.3 \mathrm{a}$ & 70.6 a-m & $72.1 \mathrm{a}-\mathrm{k}$ & $72.3 \mathrm{abc}$ & 186.4 b-e & $174.2 \mathrm{~b}-\mathrm{e}$ & $182.5 \mathrm{~b}-\mathrm{e}$ & 181.0 a-e \\
\hline 10 & Yelesen & 71.6 a-1 & 70.8 a-m & $71.0 \mathrm{a}-\mathrm{m}$ & $71.1 \mathrm{a}-\mathrm{f}$ & $170.5 \mathrm{cde}$ & $165.7 \mathrm{cde}$ & $180.7 \mathrm{~b}-\mathrm{e}$ & $172.3 \mathrm{cde}$ \\
\hline 11 & Çeltiksuyu-1 & $71.4 \mathrm{a}-1$ & $69.3 \mathrm{~g}-\mathrm{m}$ & 70.7 a-m & $70.5 \mathrm{c}-\mathrm{f}$ & $165.6 \mathrm{cde}$ & $175.0 \mathrm{~b}-\mathrm{e}$ & $179.4 \mathrm{~b}-\mathrm{e}$ & $173.4 \mathrm{~b}-\mathrm{e}$ \\
\hline 12 & Çeltiksuyu-2 & $71.1 \mathrm{a}-\mathrm{m}$ & $71.8 \mathrm{a}-\mathrm{k}$ & $72.0 \mathrm{a}-\mathrm{k}$ & 71.6 a-e & $165.7 \mathrm{cde}$ & $195.5 \mathrm{a}-\mathrm{e}$ & $183.6 \mathrm{~b}-\mathrm{e}$ & 181.6 a-e \\
\hline 13 & Çeltiksuyu-3 & 72.8 a-h & $67.7 \mathrm{~lm}$ & $70.1 \mathrm{c}-\mathrm{m}$ & $70.2 \mathrm{def}$ & $182.4 \mathrm{~b}-\mathrm{e}$ & $157.1 \mathrm{cde}$ & $143.8 \mathrm{de}$ & $161.1 \mathrm{e}$ \\
\hline 14 & Meşedal1-1 & $72.5 \mathrm{a}-\mathrm{j}$ & 69.6 e-m & 70.7 a-m & 70.9 a-f & $178.4 \mathrm{~b}-\mathrm{e}$ & $157.3 \mathrm{cde}$ & 180.7 b-e & $172.1 \mathrm{cde}$ \\
\hline 15 & Meşedal1-2 & $72.7 \mathrm{a}-\mathrm{i}$ & 70.5 a-m & $69.4 \mathrm{f}-\mathrm{m}$ & 70.9 a-f & $179.8 \mathrm{~b}-\mathrm{e}$ & $156.5 \mathrm{cde}$ & $161.1 \mathrm{cde}$ & $165.8 \mathrm{cde}$ \\
\hline 16 & Servi & 70.2 b-m & $69.9 \mathrm{c}-\mathrm{m}$ & $68.8 \mathrm{i}-\mathrm{m}$ & $69.6 \mathrm{f}$ & 160.2 cde & $159.1 \mathrm{cde}$ & $167.8 \mathrm{cde}$ & $162.4 \mathrm{de}$ \\
\hline 17 & Çevirme & $71.3 \mathrm{a}-\mathrm{m}$ & 70.6 a-m & $71.2 \mathrm{a}-\mathrm{m}$ & $71.0 \mathrm{a}-\mathrm{f}$ & $165.2 \mathrm{cde}$ & $161.1 \mathrm{cde}$ & $158.2 \mathrm{cde}$ & $161.5 \mathrm{e}$ \\
\hline 18 & Arslanbeyli & 73.5 a-e & 69.1 h-m & 70.2 b-m & 70.9 a-f & $189.5 \mathrm{~b}-\mathrm{e}$ & $160.2 \mathrm{cde}$ & $175.8 \mathrm{~b}-\mathrm{e}$ & $175.1 \mathrm{~b}-\mathrm{e}$ \\
\hline 19 & Mutluca & $73.1 \mathrm{a}-\mathrm{g}$ & $69.4 \mathrm{f}-\mathrm{m}$ & $68.3 \mathrm{klm}$ & $70.3 \mathrm{def}$ & $183.0 \mathrm{~b}-\mathrm{e}$ & 170.9 cde & $146.0 \mathrm{de}$ & $166.6 \mathrm{cde}$ \\
\hline 20 & Bağliisa & 73.6 a-d & $68.6 \mathrm{j}-\mathrm{m}$ & $68.3 \mathrm{klm}$ & $70.1 \mathrm{def}$ & 190.7 b-e & $154.5 \mathrm{de}$ & $138.5 \mathrm{e}$ & $161.2 \mathrm{e}$ \\
\hline 21 & Taşlıçay & $73.6 \mathrm{abc}$ & 70.8 a-m & $70.0 \mathrm{c}-\mathrm{m}$ & $71.5 \mathrm{a}-\mathrm{f}$ & 185.9 b-e & $170.0 \mathrm{cde}$ & 165.8 cde & $173.9 \mathrm{~b}-\mathrm{e}$ \\
\hline 22 & Şenköy-1 & $72.5 \mathrm{a}-\mathrm{i}$ & $67.5 \mathrm{~m}$ & $69.9 \mathrm{c}-\mathrm{m}$ & 70.0 ef & $178.6 \mathrm{~b}-\mathrm{e}$ & $146.2 \mathrm{de}$ & 170.5 cde & $165.1 \mathrm{de}$ \\
\hline 23 & Şenköy-2 & $72.0 \mathrm{a}-\mathrm{k}$ & 69.6 e-m & $71.9 \mathrm{a}-\mathrm{k}$ & $71.2 \mathrm{a}-\mathrm{f}$ & $177.3 \mathrm{~b}-\mathrm{e}$ & 169.8 cde & $189.7 \mathrm{~b}-\mathrm{e}$ & 178.9 a-e \\
\hline 24 & Başbağ & $71.9 \mathrm{a}-\mathrm{k}$ & $73.1 \mathrm{a}-\mathrm{g}$ & $71.0 \mathrm{a}-\mathrm{m}$ & 72.0 a-d & $175.8 \mathrm{~b}-\mathrm{e}$ & $232.5 \mathrm{ab}$ & $166.8 \mathrm{cde}$ & $191.7 \mathrm{a}-\mathrm{d}$ \\
\hline 25 & Bilensoy 80 & 72.9 a-h & $74.0 \mathrm{ab}$ & 71.0 a-m & $72.6 \mathrm{ab}$ & $181.2 \mathrm{~b}-\mathrm{e}$ & $251.0 \mathrm{a}$ & $174.4 \mathrm{~b}-\mathrm{e}$ & $202.2 \mathrm{ab}$ \\
\hline 26 & Gea & $72.8 \mathrm{a}-\mathrm{h}$ & $74.3 \mathrm{a}$ & 70.9 a-m & $72.6 \mathrm{ab}$ & 184.9 b-e & $253.7 \mathrm{a}$ & $185.9 \mathrm{~b}-\mathrm{e}$ & $208.1 \mathrm{a}$ \\
\hline \multirow[t]{4}{*}{27} & Savaş & $69.8 \mathrm{c}-\mathrm{m}$ & 72.5 a-j & $71.3 \mathrm{a}-\mathrm{m}$ & $71.2 \mathrm{a}-\mathrm{f}$ & $159.4 \mathrm{cde}$ & $215.4 \mathrm{abc}$ & $190.5 \mathrm{~b}-\mathrm{e}$ & 188.4 a-e \\
\hline & Mean & $71.9 \mathrm{~A}$ & $70.5 \mathrm{~B}$ & $70.5 \mathrm{~B}$ & 71.0 & 173.1 & 177.1 & 171.4 & 173.8 \\
\hline & $\operatorname{LSD}(0.05)$ & \multicolumn{4}{|c|}{ Year (Y):0.41*, Genotype (G): $1.97^{*}$, Y x G : 3.90* } & \multicolumn{4}{|c|}{$\begin{array}{l}\text { Year (Y): not significant, Genotype (G): } 29.9^{* *}, \text { Y x G } \\
: 59.2^{* *}\end{array}$} \\
\hline & & \multicolumn{4}{|c|}{$\mathrm{CV}(\%): 1.56,{ }^{* *} \mathrm{P} \leq 0.01$} & \multicolumn{4}{|c|}{$\mathrm{CV}: \% 9.69,{ }^{* *} \mathrm{P} \leq 0.01$} \\
\hline
\end{tabular}

year, and at Kumgecit population for the average of three years $(72.7 \%)$.

2016, 2017, 2018 and three years average RFV of average of all genotypes were 173.1, 177.1, 171.4 and 173.8 , respectively. 8 of 27 genotypes were yielded high and positioned in the same statistical group according to three years average RFV values. RFV values were highest at Bagliisa population in the first year (190.7), at Gea variety in the second year (253.7), at Kumgecit population (199.4) in the third year, and at Gea variety for the average of three years (208). In a study of BASBAG et al., (2017), 16 individual plants were selected from the Southeastern Turkey from their natural habitat and clonally propagated in field trials where relative feed value ranged from 134.3 to 306.1 .
The obtained DDM rates were similar to the findings of CACAN et al. (2018) (71.0-74.4\%). On the other hand, our RFV values were higher than GUNDEL et al. (2014) and ENGIN \& MUT (2018), and lower than CACAN et al. (2018). BASBAG et al. (2009), collected 11 alfalfa clones from Southeastern and Eastern Regions of Turkey and determined a significant variation in quality properties of alfalfa clones for DDM (63.0-75.8\%). Since the relative feed value is calculated from the ADF and NDF ratios, the difference between these two values changes the relative feed value significantly. In general, the alfalfa plant has an excellent RFV quality grade (170-180 RFV) when it contains $21-22 \% \mathrm{CP}$, less than $28 \%$ ADF and less than 35\% NDF (BOMAN, 2017). When these characteristics are considered together, herbage 
quality of alfalfa genotypes that we examined in the study are in excellent grade.

\section{CONCLUSION}

The natural variation in wild germplasm is very wide and could be detected and transferred in breeding programs and this variation has been reported earlier in Turkish alfalfa germplasm (SAKIROGLU et al. 2011). The region that genotypes collected in our study is in the Fertile Crescent and also in the intersection of Vavilov's two centers of origins, Transcaucasia and Mediterranean. The region has been noted to be one of the centers of diversity for alfalfa (SMALL, 2011; SAKIROGLU \& BRUMMER 2013). Germplasm collected showed a desirable charecters for improving yield and quality of alfalfa. Our results indicated that the collection and utilization of wild alfalfa germplasm is crucial for increasing its adaptation. In terms of both green herbage and hay yield, Servi, Mutluca, Garip,Sarıçiçek-1, Bagliisa village populations were found superior to registered varieties. When we compare the quality results, Kumgecit, Küçüktekören and Şenköy-1 populations stands out in terms of crude protein ratio. Highest crude protein yield was obtained from Servi population. In terms of relative feed value, it was observed that the varieties were better qualfied than the populations. It is concluded that the populations of Servi, Mutluca, Garip, Sarıçiçek-1 and Bagliisa can be used in breeding studies especially to obtain genetic progress in yield of alfalfa for feed and bioenergy production. For the genetic improvement of current genotypes with higher crude protein ratio, Kumgecit, Küçüktekören and Şenköy-1 populations can be used as a source.

\section{ACKNOWLEDGEMENTS}

We would like to thank our rector Ibrahim ÇAPAK, Bingöl University, who supported us in carrying out this study.

\section{DECLARATION OF CONFLICT OF INTERESTS}

The authors declare no conflict of interest. The founding sponsors had no role in the design of the study; in the collection, analyses, or interpretation of data; in the writing of the manuscript, and in the decision to publish the results.

\section{AUTHORS' CONTRIBUTIONS}

All authors contributed equally for the conception and writing of the manuscript. All authors critically revised the manuscript and approved of the final version.

\section{REFERENCES}

ANONYMOUS, Turkish state meteorological service. Available from: $<$ https://mgm.gov.tr/eng/forecast-cities.aspx $>$. Accessed: Jan. 20, 2019

AVCIOGLU, R. et. al. Alfalfa (Medicago sp. L.). (Ed. R. Hatipoglu, Y. Karadag), forage crops C-II: legume forage crops, General Directorate of Agricultural Research and Policies, Izmir, p.293295. 2009. Available from: <https://www.kitantik.com/product/ YEMBITKILERI-3-CILT-TAKIM_0z8kgltj7d3aput $1 \mathrm{bx} 0>$. Accessed: Jan. 20, 2019.

BASBAG, M., et al. Determination of some agronomical and quality properties of wild alfalfa (Medicago sativa L.) clones in Turkey. Journal of Food, Agriculture \& Environment, v.7, n.2, p.357-359 2009. Available from: <https://www.researchgate.net/ publication/236151733_Determination_of_some_agronomical_ and_quality_properties_of_wild_alfalfa_Medicago_sativa_L_ clones_in_Turkey>.Accessed: Feb. 25, 2019.

BASBAG, $M$. et al. Evaluating agronomic performance and investigating molecular structure of drought and heat tolerant wild alfalfa (Medicago sativa L.) collection from the Southeastern Turkey. Biochemical Genetics, v.55, n.1, p.6376. 2017. Available from: <https://www.semanticscholar.org/ paper/Evaluating-Agronomic-Performance-and-Investigating$\mathrm{Ba} \% \mathrm{C} 5 \% 9 \mathrm{Fba} \% \mathrm{C} 4 \% 9 \mathrm{~F}-A y d i n / 48$ ed 40 cf5ef55c9e 9 be $69 \mathrm{cb} 4 \mathrm{~d} 040$ 2583eb73a563>. Accessed: Feb. 25, 2019. doi: 10.1007/s10528016-9772-7.

CACAN, E. et al. Determination of yield and quality characteristics of some alfalfa (Medicago sativa L.) cultivars in the east anatolia region of turkey and correlation analysis between these properties. Applied Ecology and Environmental Research v.16, n.2, p.1185-1198. 2018. Available from: <http://www.aloki. hu/indvol16 2.htm>. Accessed: Mar. 07, 2019. doi: 10.15666/ aeer/1602_11851198.

DEMIROGLU, G.; AVCIOGLU, R. An investigation on the performances of some new leguminous forage crops cultivars under mediterranean climatic conditions. Journal of Agriculture Faculty of Ege University, v.47 n.2, p.151-159. 2010. Available from: <https://dergipark.org.tr/tr/pub/zfdergi/issue/5097/69609>. Accessed: Mar. 13, 2019.

DIEN, B. S. et al. Chemical composition and response to diluteacid pretreatment and enzymatic saccharification of alfalfa, reed canarygrass, and switchgrass. Biomass and Bioenergy, v.30, n.10, p.880-891. 2006. Available from: $<$ https://www.sciencedirect.com/ science/article/pii/S0961953406000547>. Accessed: May, 25, 2019. doi: 10.1016/j.biombioe.2006.02.004.

ENGIN, B.; MUT, H. Determination of hay yield and some quality traits of different alfalfa cultivars. Yuzuncu Yıl University Journal of Agricultural Sciences, v.27, n.2, p.212-219. 2017. Available from: <https://dergipark.org.tr/tr/pub/yyutbd/ issue/30352/289466>. Accessed: May, 13, 2019. doi: 10.29133/ yyutbd.289466.

ENGIN, B.; MUT, H. Variation of some nutrient contents with relative feed value according to cutting order in alfalfa (Medicago sativa L.) varieties. Journal of Tekirdag Agricultural Faculty, v.15, n.2, p.119-127. 2018. Available from: <https://dergipark.org. tr/tr/pub/jotaf/issue/37314/293214>. Accessed: May, 13, 2019. 
GOKALP, S. et al. Determination of forage yield and quality performance of some alfalfa (Medicago sativa L.) cultivars in Tokat-Kazova ecological conditions. Journal of Agricultural Faculty of Gaziosmanpasa University, v.34, n.3, p.114127. 2017. Available from: <http://ziraatdergi.gop.edu.tr/ Makaleler/292925579_114-127.pdf>. Accessed: Feb. 02, 2019. doi: $10.13002 /$ jafag4332.

GUNDEL, F. D. et al. A Research on yield, quality and adaptation of some warm season perennial legumes under cukurova ecological conditions. Journal of Agricultural Faculty of Gaziosmanpasa University, v.31, n.3, p.10-19. 2014. Available from: <http://ziraatdergi.gop.edu.tr/Makaleler/947806780_10-19. pdf $>$. Accessed: Mar. 20, 2019.

JAFARI, A.; RAZBAN, H. A. Evaluation of forage yield and quality of alfalfa (Medicago sativa) accessions in dryland conditions of Eastern Azerbaijan. Plant Ecophysiology, (Arsanjan Branch) v.10, n.32, p.94-107. 2018. Available from: $<$ https://www.sid.ir/en/journal/ViewPaper.aspx?ID=667805> Accessed: Mar. 13, 2019.

JULIER, B. et al. Within-and among-cultivar genetic variation in alfalfa: forage quality, morphology, and yield, Crop Science, v.40 n.2, p.365-369. 2000. Available from: <https://www. researchgate.net/publication/240610400 Within and AmongCultivar_Genetic_Variation_in_Alfalfa_Forage_Quality Morphology_and_Yield $>$. Acessed: Mar. 25, 2019. doi: 10.2135/ cropsci2000.402365x.

KARAKOY, T.; SARAC, H. Determination of some agronomical and quality properties of alfalfa (Medicago sativa L.) cultivars in sivas ecological conditions.Turkish Journal of Agricultural and Natural Sciences, v.5, n.4, p.620-627. 2018. Available from: $<$ https://dergipark.org.tr/tr/pub/turkjans/issue/39809/471373>. Accessed: Apr. 25, 2019. doi: 10.30910/turkjans.471373.

KAVUT, Y. T. et al. An Investigation on yield and some yield characteristics of different alfalfa genotypes grown in different locations. Journal of Agriculture Faculty of Ege University, v.51, n.1, p.23-29. 2014. Available from: <https://dergipark.org. tr/tr/download/article-file/59462>. Accessed: Apr. 20, 2019. doi: $10.20289 /$ euzfd. 89017 .

LI, X.; Brummer, E.C. Applied genetics and genomics in alfalfa breeding. Agronomy, v.2, n.1, p.40-61. 2012. Available from: $<$ https://www.mdpi.com/2073-4395/2/1/40>. Accessed: May, 15, 2019. doi: 10.3390/agronomy 2010040 .

MARTIN, N. P.; Jung, H. J. C. Alfalfa: potential for new feed and biofuel-usdfrc research update. Winter Seed School Conference, Golden Nugget, Las Vegas, NV, January 17-19, 2010. p.105-111. Available from: <https://pdfs.semanticscholar. org/b06d/a 5144 a 0 caffoaf 156 ea 1 b 651 e 10 be 5 a 5 d 8 c 7 . pdf? ga $=2.258548490 .1889915818 .1594629639$. 1210075223.1594629639>. Accessed: May, 13, 2019.

MORRISON, J. A. Hay and Pasture Management, Chapter 8. Extension Educator, Crop Systems Rockford Extension Center, 2003. Available from: <http://iah.aces.uiuc.edu/pdf/Agronomy HB/ 08chapter.pdf $>$. Accessed: Jun. 13, 2019.

OTEN, M.; ALBAYRAK, S. Determination of forage quality features of some alfalfa (Medicago sativa L.) genotypes. Journal of Field Crops Central Research Institute, v.27, n.2, p.55-61 2018. Available from: <https://dergipark.org.tr/tr/pub/tarbitderg/ issue/41806/501415>. Accessed: Jun. 13, 2019. doi: 10.21566/ tarbitderg.501415.

PUTNAM, D. et al. Alfalfa, wildlife and the environment. California Alfalfa and Forage Association, Novato, CA. Available from: $<$ https://www.alfalfa.org/pdf/alfalfaenvironment2.pdf $>$. Accessed: Jun. 13, 2019

RIDAY, H.; BRUMMER, E. C. Forage yield heterosis in alfalfa. Crop Science, v.42 n.3, p.716-723. 2002. Available from: $<$ https://acsess. onlinelibrary.wiley.com/doi/pdf/10.2135/cropsci2002.7160>. Accessed: Jun. 23, 2019.

SAKIROGLU, M.; BRUMMER, E. C. Presence of phylogeographic structure among wild diploid alfalfa accessions (Medicago sativa L. subsp. microcarpa Urb.) with evidence of the center of origin. Genetic Resources and Crop Evolution, v.60, n.1, p.23-31. 2013. Available from: <https:/www.researchgate.net/ publication/257567628_Presence_of_phylogeographic_structure among_wild_diploid_alfalfa_accessions_Medicago_sativa_L_ subsp_microcarpa_Ürb_with_evidence_of_the_center_of origin $>$. Acessed: Jul. $13, \overline{2} 019$. doi: $10.1007 / \mathrm{s} 10722-012-9811-0$.

SAKIROGLU, M. et al. Variation in biomass yield, cell wall components, and agronomic traits in a broad range of diploid alfalfa accessions. Crop Science, v.51, n.5, p.19561964. 2011. Available from: <https:/www.researchgate.net/ publication/259779589 Variation in Biomass Yield Cell Wall_Components_and_Agronomic_Traits_in_a_Broad_Range_ of_Diploid_Alfalfa_Accessions $>$. Accessed: Jul. 13, 2019. doi: 10.2135 /cropsci2010.11.0658.

SARUHAN, V.; KUSVURAN, A. Determination of Yield performances of some lucerne cultivars and genotypes under the southeastern anatolia region conditions. Journal of Agriculture Faculty of Ege University, v.48, n.2, p.133-140. 2011. Available from: <https://dergipark.org.tr/tr/pub/zfdergi/issue/5101/69647>. Accessed: Jul. 13, 2019.

SEKER, H. Adaptation and yield trial of some new alfalfa cultivars to erzurum ecological condition. Atatürk University Journal of Agricultural Faculty, v.34, n.3, p.217-221. 2003. Available from: $<$ https://dergipark.org.tr/tr/pub/ataunizfd/issue/2944/40743>. Accessed: Jul. 13, 2019.

SENGUL, S. et al. Determination of suitable alfalfa (Medicago sativa $\mathrm{L}$ ) cultivars and lines for Eastern Anatolia Region. Atatürk University Journal of Agricultural Faculty, v.34, n.4, p.321-325. 2003. Available from: <https://dergipark.org.tr/tr/pub/ataunizfd/ issue/2939/40728>. Accessed: Jun. 13, 2019.

SEZEN, Y. Fertilizers and Fertilization. Ataturk University Publication No:679, Agriculture Faculty Publications, No:303, s.15, Erzurum. 1995. Available from: <https://books.google.com. tr/books?id=1TXBq1IbMtgC\&pg=PA135\&lpg=PA135\&dq=Fer tilizers + and + Fertilization. + Ataturk + University + Publication\&so urce $=$ bl\&ots $=$ SAx2a7I3 vw\&sig=ACfU3U2GAPHBrObXcR6y pi87biTEEA5gYw\&hl $=$ tr\&sa $=$ X\&ved $=2$ ahUKEwju3unSjsrqAh WClosKHasCAWwQ6AEwC3oECAcQAQ\#v=onepage\&q=Fer tilizers\%20and\%20Fertilization.\%20Ataturk\%20University\%20 Publication\&f=false $>$. Accessed: Apr. 25, 2019.

SHEAFFER, C. C. et al. Leaf and stem properties of alfalfa entries. Agronomy Journal, v.92, n.4, p.733-739. 2000. Available from: <https://acsess.onlinelibrary.wiley.com/doi/epdf/10.2134/ agronj2000.924733x>. Accessed: May, 25, 2019. 
SMALL, E. Alfalfa and relatives: evolution and classification of medicago. CAB Intl, Wallingford. 2011. Available from: <https:// www.nrcresearchpress.com/doi/book/10.1139/9780660199795\#. XwxECCgzaUk>. Accessed: Apr. 25, 2019.

SOYA, H. et al. Forage crops. Hasat Publication, Istanbul, p.131. 2004. Available from: <https://www.hasad.com.tr/>. Accessed: May, 15, 2019.

TURAN, N. Yield and quality performances of alfalfa (Medicago sativa) cultivars sown at various dates under sub-mediterranean ecological conditions. Applied Ecology and Environmental
Research, v.17, n.6, p.15615-15631. 2019. Available from: $<$ http:// www.aloki.hu/indvol17_6.htm>. Accessed: Jul. 13, 2020. doi: 10.15666/aeer/1706_1561515631.

TURAN, N. et al. Yield and quality characteristics of some alfalfa(Medicago sativa $\mathrm{L}$ ) varieties grown in the eastern Turkey. Turkish Journal of Field Crops, v.22, n.2, p.160-165. 2017. Available from: <https://dergipark.org.tr/tr/download/articlefile/368041>. Accessed: Jul. 13, 2019. doi: 10.17557/tjfc.356236.

TURKSTAT. Statistical Institute of Turkey, Crop Production Statistics. Available from: < http://www.tuik.gov.tr>. Accessed: Jul. 13, 2019. 